\title{
Whole body and regional fat and lean mass in Bulgarian women of different ages and body mass index
}

\author{
Mihail A Boyanov
}

Clinic of Endocrinology and Metabolic Diseases, University Hospital Alexandrovska, Department Internal Medicine, Faculty of Medicine, Medical University of Sofia, Bulgaria

Background: Obesity has become a worldwide epidemic. $38.8 \%$ of men and $28.3 \%$ of the women in a recent Bulgarian population study had overweight or obesity. ${ }^{1}$ Body composition analysis provides useful information in epidemiological studies of obesity and overweight, as well as of sarcopenia and frailty. ${ }^{2}$ Dual-energy X-ray absorptiometry (DXA) is a reliable method for body composition analysis. ${ }^{3}$ The International Society for Clinical Densitometry (ISCD) 2013 Conference advocated the use of whole body scans in specific clinical situations. ${ }^{4} \mathrm{~A}$ number of studies have published data from various reference populations - Europeans, Asians and Americans. ${ }^{5-9}$ The

\section{Subjects and Methods}

This is a post hoc analysis of data coming from a cross-sectional study in a hospital-based outpatient DXA setting ${ }^{11}$. A total of 159 women participated in the original study but only 140 were suitable for proper analysis. Three subgroups were built according to age: 30 women aged 20-44 years, 80 women aged $45-59$ years and 30 women aged 60-75 years. 72 of the women were referred for bone density testing (65 postmenopausal and 7 premenopausal women), 56 - for body composition analysis because of overweight or obesity (22 premenopausal and 34 postmenopausa women) and 12 were healthy volunteers (1 premenopausal and 11 postmenopausal women). All women had given their informed consent prior to any procedure.

Body composition analysis

The subjects were required to adhere to standard body composition testing guidelines, wearing light clothes. The measurements were performed on a Hologic QDR 4500 A bone densitometer (Hologic Inc., Bedford, MA 02154, USA). All scans were read by the same technologist

NHANES 1999-2004 database is an effort to provide reliable and internationally validated reference values for body composition analysis. ${ }^{5}$ The ISCD 2013 Conference recommends using them instead of manufacturer-provided reference populations. ${ }^{10}$

The aim of the present study was to perform total and sub-regional body composition analysis in Bulgarian women aged 20-75 years with normal weight and different levels of overweight/obesity and to calculate age-related means for FM and LM and \% FM.

with software version 8.26:3. The data were presented as FM in grams, LM in grams, and bone mineral content (BMC) in grams. The percentage of $\mathrm{FM}(\% \mathrm{FM})$ was also calculated. Data were calculated separately for the different body sub-regions (arms, legs and trunk) as well as subtota (excluding the head) and total values.

Precision study

The in vivo CV \% of DXA was $0.79 \%$ for \% FM, 760 grams for FM (corresponding to $2.05 \%$ ), 710 grams for LM $(1.46 \%)$ and 40 grams for BMC $(2.01 \%)$. The mean difference between body weight coming from DXA and from the digital scale balance in our dataset was below $0.5 \mathrm{~kg}$, meaning that DXA had quite accurately estimated real weight.

Statistical analysis

An IBM SPSS 19.0 for WINDOWS package (Chicago, IL, USA) was used. Statistical significance was set as $\mathrm{p} \leq 0.05$.

Results

The anthropometric and whole body composition data of the participants are presented in Table 1. Total body FM declined steadily with increasing age, with a steeper decline in the elderly group. Total body LM, on the contrary, showed a more pronounced difference between age groups 20-44 and 45-59 years than between $45-59$ and 60-75 years.

Table 1. Anthropometric and body composition data of the study population are shown as means (standard deviations). Total bone mineral content (BMC) as well as $L M$ and FM are presented in kilograms, bone mineral density (BMD) in grams per centimeters squared.

Fig. $1 \mathrm{~A}-\mathrm{C}$ show the age-adjusted means of FM and LM in three different body regions. In the arms LM shows a greater difference between 20-44 and 45-59 years of age than between 45-59 and 60-75 years, while the difference in FM is greater between ages $45-59$ and $60-75$ years (Fig. 1A). The

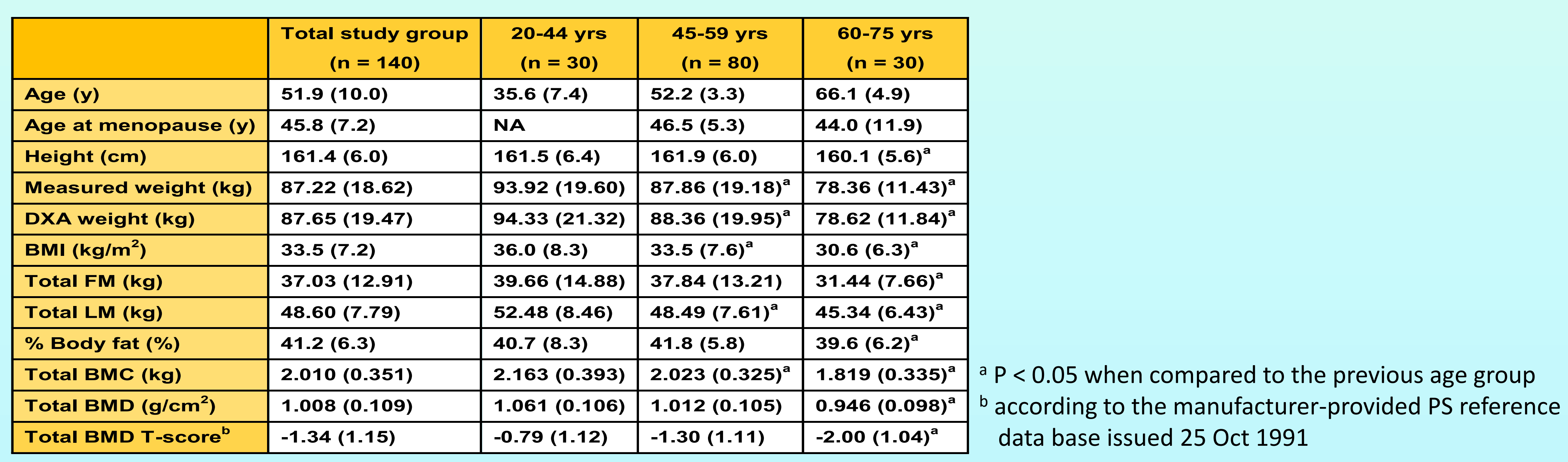

same tendency of FM declining more steeply in older ages can be seen in the trunk (Fig. 1C), while in the legs FM and LM show an almost parallel decline with age (Fig. 1B).

Figure 1. The age-adjusted means of fat and lean mass (in kilograms) in three different body regions - arms, legs and trunk, are shown.
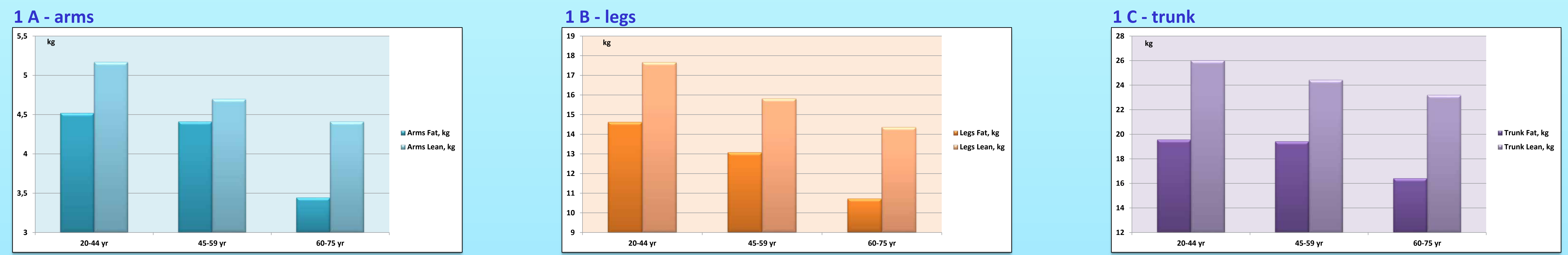

The mean percentage of total and regional FM is displayed in Fig. 2. It is highest in the middleaged (45-59 years) group.

Fig. 3 shows the ratio of appendicular to trunk fat and lean mass in the different age groups.

Figure 2. The mean percentage of total and regional (arms, legs, trunk) \% FM is displayed.

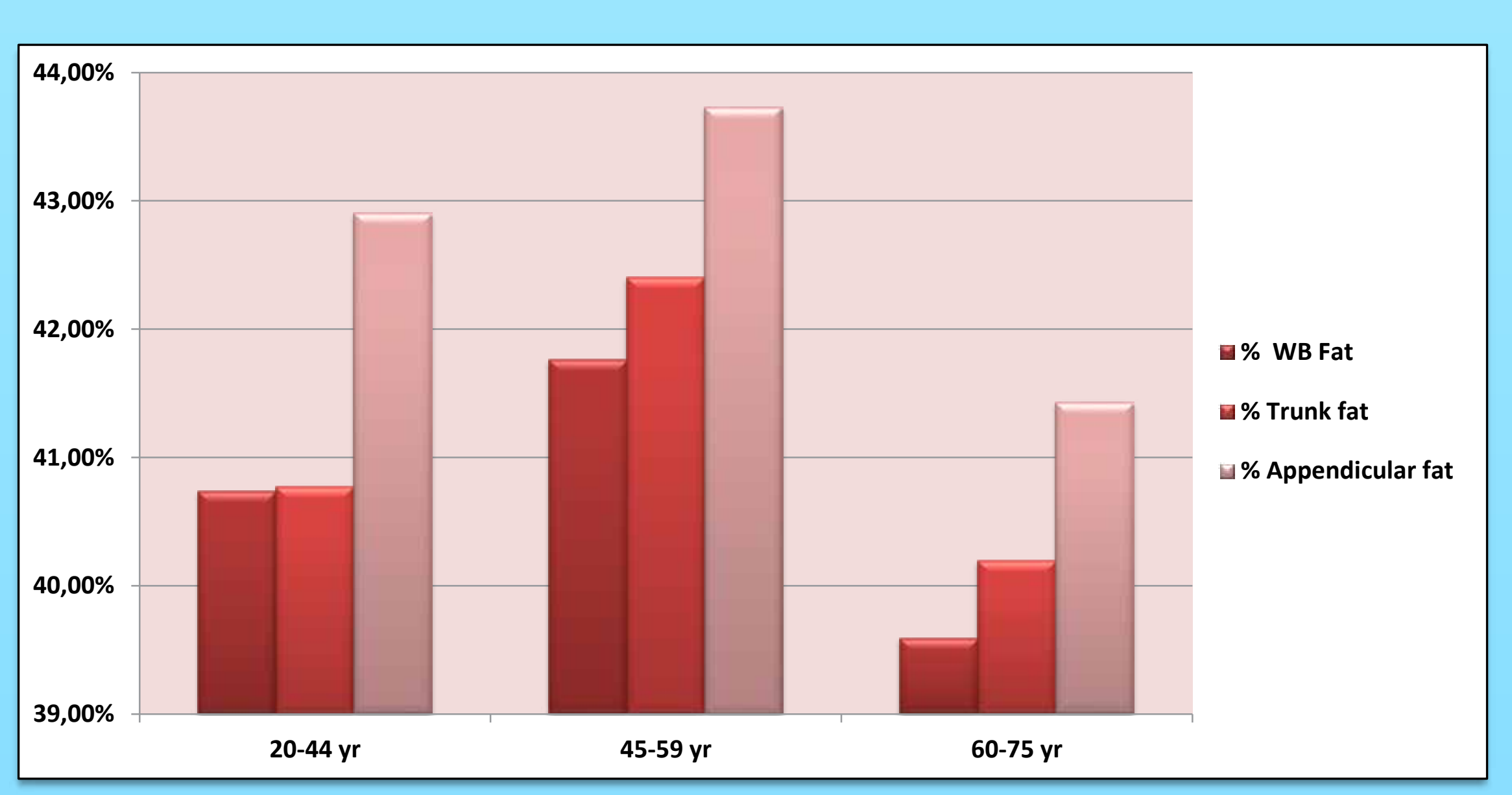

The proportion is decreasing steadily with age showing a more centripetal body mass predisposition The most marked redistribution can be seen between 20-44 and 45-59 years involving fat mass.

igure 3. Ratio of appendicular to trunk fat and lean mass in the different age groups

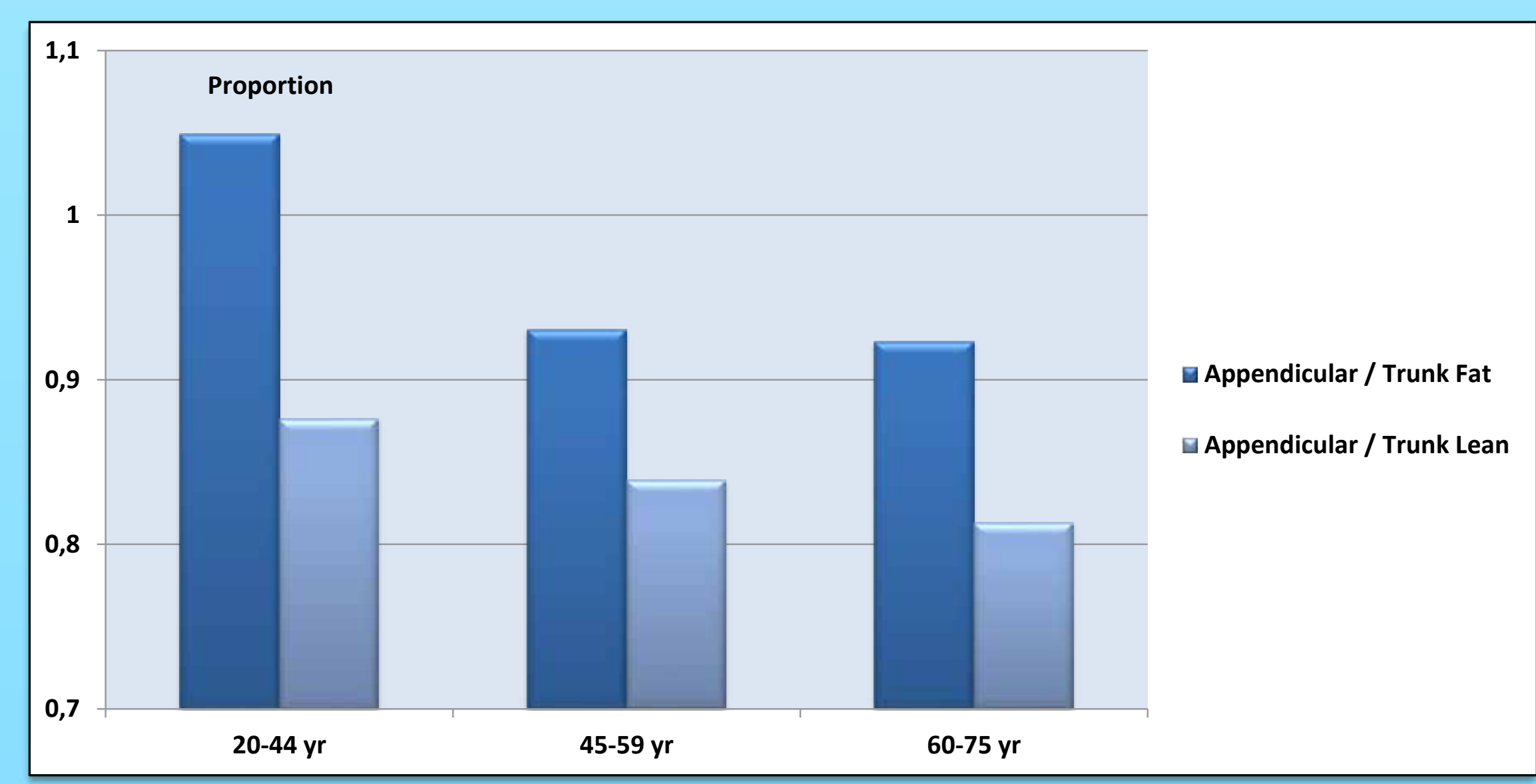

Discussion

This is the first Bulgarian study reporting DXA-derived estimates of whole and regional body composition analysis. FM was lost at different rates in the trunk, legs and arms and predominantly in older ages whereas LM followed an almost linear pattern of decline with age. This resulted in \% FM being highest in the middle-aged group (45-59 yrs.). Trunk fat remained almost similar in age groups 20-44 and 45-59 yrs which led to a steep decrease of the proportion of appendicular / trunk fat. We tried to explain these findings by at least three factors: 1 / increasing chronological age; $2 /$ the perimenopausal and postmenopausal transition, as all women under 45 were premenopausal, while those elderly were postmenopausal; 3 / the decreasing BMI in the elderly age groups leading to smaller body proportions affecting the FM and LM and their ratio in the trunk and limbs.

Our study has some limitations. First, the number of participants is rather modest which prevented us from separate sub-analyses based on BMI or menopausal status. Second, this is a post hoc analysis based on a sample referred for DXA and our data are not generalizable. And third, the study design is cross-sectional, thus allowing rough estimates of real age-related changes in body composition.

The major strength of this study is that it is the first one in our country looking for BMC and BMD changes assessed by DXA. It showed differing patterns of age-related decline in regional FM and $L M$, It displays some directions for future research in the assessment of sarcopenia and body composition in different female populations.

Acknowledgments: The author would like to thank Assoc. Prof. Dr. Zhivka Boneva-Asiova (Head of the Endocrine Unit, MVR Institute - Central Hospital, Sofia) and the DXA technologist Darina Antonova from the same Endocrine Unit. Conflict of Interest: No financial support or competing financial interests in relation to this work. References

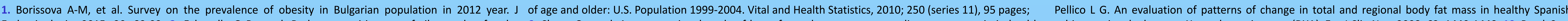

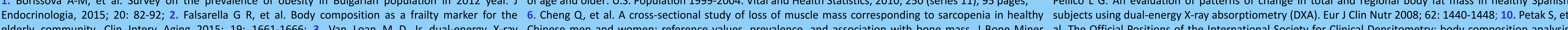

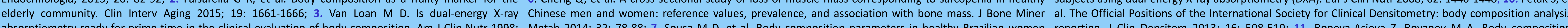

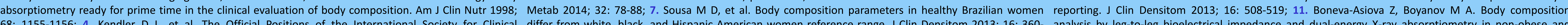
68: 1155-1156; 4. Kendler D L, et al. The Official Positions of the International Society for Clinical differ from white, black, and Hispanic American women reference range. I Clin Densitom 2013; $16: 360$.

496-507; 5. U.S. Department of Health and Human Services. Body composition data for individuals 8 years to 80-year-old healthy Italian population. Ann Nutr Metab 2012; 61: 151-9; 9. Henche S A, Torres R R,

obese individuals. Diabetes Obes Metab 2008; 10: 1012-1018.

Correspondence: Prof. Mihail Boyanov, Clinic of Endocrinology and Metabolism, University Hospital Alexandrovska, Medical University Sofia; 1, G. Sofiyski street, 1431 Sofia, Bulgaria; e-mail: mihailboyanov@yahoo.com 